Pacific Journal of Mathematics

A STUDY OF REGULARITY PROBLEM OF HARMONIC MAPS 


\section{A STUDY OF REGULARITY PROBLEM OF HARMONIC MAPS}

\section{GuOJUN LiaO}

In this paper we study the regularity problem of harmonic maps between closed compact manifolds $\left(M^{n}, g\right)$ and $\left(N^{m}, h\right)$ in dimensions $n \geq 3$.

1. Introduction. Harmonic maps are critical points of the energy functional. For technical convenience we assume, by virtue of the Nash imbedding theorem, that the target manifold $N$ is isometrically imbedded in the smallest Euclidean space $\mathbf{R}^{k}$. At the end of this section we will discuss the independence of our definitions on the imbedding of $N$ in $\mathbf{R}^{k}$.

Definition (1.1). A map $u=\left(u^{1}, u^{2}, \ldots, u^{k}\right): M \rightarrow \mathbf{R}^{k}$ is said to belong to the Sobolev space $L_{1}^{2}\left(M, \mathbf{R}^{k}\right)$ if for $i=1,2, \ldots, k$

$$
\int_{M}\left|\nabla u^{i}\right|^{2} d V<\infty
$$

where $\left|\nabla u^{i}\right|$ is the covariant derivative, in local coordinates,

$$
\left|\nabla u^{\prime}\right|^{2}(x)=g^{\alpha \beta}(x) \cdot \partial_{\alpha} u^{i} \cdot \partial_{\beta} u^{l},
$$

$d V$ is the volume element of $M$. For $u \in L_{1}^{2}\left(M, \mathbf{R}^{k}\right)$ one defines its energy as

$$
E(u)=\sum_{i=1}^{k} \int_{M}\left|\nabla u^{i}\right|^{2} d V=\int_{M}|\nabla u|^{2} d V .
$$

Definition (1.2). A map $u$ is said to belong to $L_{1}^{2}(M, N)$ if $u \in$ $L_{1}^{2}\left(M, \mathbf{R}^{k}\right)$ and if $u(x) \in N$, a.e. $x \in M$.

REMARK. $L_{1}^{2}\left(M, \mathbf{R}^{k}\right)$ with the usual norm

$$
|u|_{1,2}=\left(E(u)+\sum_{l=1}^{k} \int_{M}\left|u^{\imath}\right|^{2} d V\right)
$$

is a separable Hilbert space. $L_{1}^{2}(M, N)$ has strong and weak topologies induced from that of $L_{1}^{2}\left(M, \mathbf{R}^{k}\right)$. Moreover, the set

$$
\left\{u \in L_{1}^{2}(M, N):|u|_{1,2} \leq C\right\}
$$

is weakly compact in $L_{1}^{2}\left(M, \mathbf{R}^{k}\right)$. 
Definition (1.3). A weak solution $u \in L_{1}^{2}(M, N)$ to the formal Euler-Lagrange equations of the energy functional is called a harmonic map from $M$ into $N$. The equations in local coordinates form a nonlinear elliptic system

$$
\Delta u^{l}(x)=g^{\alpha \beta}(x) \cdot A_{u(x)}^{i}\left(\partial_{\alpha} u, \partial_{\beta} u\right), \quad i=1,2, \ldots, k,
$$

where $A_{u}(X, Y) \in\left(T_{u} N\right)^{\perp}$ for vectors $X, Y \in T_{u} N$ is the second fundamental form of $N$ given by

$$
A_{u}(X, Y)=\left(D_{X} Y\right)^{\perp},
$$

$X$ and $Y$ are considered to have been extended to vector fields on $N$ in a neighborhood of $u \in N$.

It is easy to see that $u$ is harmonic if and only if

$$
\left.\frac{d}{d t}\right|_{t=0} E\left(u_{t}\right)=0
$$

where $u_{t}$ is a 1-parameter family of maps defined by

$$
\begin{gathered}
u_{t}(x)=\Pi\left(u(x)+\eta_{t}(x)\right), \\
\eta_{t} \in C_{0}^{\infty}\left(M, \mathbf{R}^{k}\right), \quad t \in[0,1] .
\end{gathered}
$$

$\Pi$ is the orthogonal projection of $\mathbf{R}^{k}$ into $N$. Next we introduce the concept of stationarity. One takes $u_{t}=u \cdot \phi_{t}$ for $\phi_{t}$ a 1-parameter family of compactly supported $C^{1}$ diffeomorphisms of $M$ with $\phi_{0}=$ Id. Clearly $E\left(u_{t}\right)$ is differentiable in $t$. If $u$ is critical for all variations of this type and if $u$ is harmonic then $u$ is called a stationary map.

By our definitions a stationary map is harmonic. The converse is not known. What is known to be true is that a $C^{2}$ harmonic map is stationary. One of the properties enjoyed by stationary maps is the monotonicity formula. We will show that the monotonicity formula still holds under some assumptions about the singular set of harmonic maps.

In this paper we will study stationary maps whose singular set is of codimension greater than 2 . Our main theorem is

THEOREM. Suppose $u$ is a stationary map, whose singular set is contained in the graph of a $C^{1, \alpha}$ function with dimension $d<n-2$. There exists an $\varepsilon>0$ such that $u$ is regular if $E(u) \leq \varepsilon$.

The main theorem will be proved by a blow up argument which seems to work well for general elliptic functionals of quadratic type (cf. [Kin, Har, Lin]). 
Schoen and Uhlenbeck (1982) developed a statisfying regularity theory for energy-minimizing harmonic maps. They showed that the singular set of an energy minimizing map has codimension bigger than 2. For general harmonic maps, it was shown in [Liao] that the isolated singularities are removable if the total energy is small. In the case $n=2$, the result was due to Sacks and Uhlenbeck (1981).

To conclude this introduction we remark on the independency of our harmonic map definition on the imbedding of $N$ in $\mathbf{R}^{k}$.

Suppose there is another isometric imbedding $\tilde{N}$ in $\mathbf{R}^{k}$. The situation is this: we have (weakly)

$$
\Delta u^{i}(x)=g^{\alpha \beta}(x) \cdot A_{u(x)}^{i}\left(\partial_{\alpha} u, \partial_{\beta} u\right) \quad i=1,2, \ldots, k .
$$

We want to show that the same system is satisfied by $\tilde{u}=h \circ u$, where $h$ is a smooth isometry from $N$ onto $\tilde{N}$.

Extend $h$ arbitrarily to a smooth map from an open neighborhood of $N$ into $\mathbf{R}^{k}$. We compute that (in the weak sense)

$$
\begin{aligned}
\Delta \tilde{u}^{i}(x) & =g^{-1 / 2} \partial_{\alpha}\left(g^{-1 / 2} g^{\alpha \beta} \partial_{\beta}\left(h^{i}(u(x))\right)\right) \\
& =g^{-1 / 2} \partial_{\alpha}\left(g^{-1 / 2} g^{\alpha \beta} \partial_{\beta} u^{j} \partial_{j} h^{i}\right) \\
& =\partial_{j} h^{i} \cdot \Delta u^{j}(x)+g^{\alpha \beta} \cdot \partial_{\beta} u^{j} \cdot \partial_{l} \partial_{j} h^{i} \cdot \partial_{\alpha} u^{l} \\
& =g^{\alpha \beta} \cdot\left(\partial_{j} h^{i} \cdot A^{j}\left(\partial_{\alpha} u, \partial_{\beta} u\right)+\partial_{\beta} u^{j} \cdot \partial_{l} \partial_{j} h^{i} \cdot \partial_{\alpha} u^{l}\right) .
\end{aligned}
$$

Consider $X, Y \in T_{u(x)} N$. One can easily check that

$$
\tilde{A}(D h(X), D h(y))=D h(A(X, Y))+D^{2} h(X, Y)
$$

where $D$ is the usual differentiation in $\mathbf{R}^{k}, \tilde{A}$ is the second fundamental form of $\tilde{N} \subset \mathbf{R}^{k}$. Indeed, we have

$$
D^{2} h(X, Y)=X Y h-\left(D_{X} Y\right) h .
$$

By a rigid motion in $\mathbf{R}^{k}$, we may assume $d h(X)=X, d h(Y)=Y$, denoting by $\tilde{\nabla}$ the covariant derivative in $\tilde{N}$, by $\nabla$ the covariant derivative in $N$. We have

$$
\tilde{A}(D h(X), D h(Y))=X Y h-\left(\tilde{\nabla}_{X} Y\right) h .
$$

Subtracting (1.5) from (1.6), we get

$$
\tilde{A}(D h(X), D h(Y))=D^{2} h(X, Y)+\left(D_{X} Y-\tilde{\nabla}_{X} Y\right) h .
$$


Notice that $\tilde{N}$ and $N$ are isometric. We can replace $\tilde{\nabla}_{X} Y$ by $\nabla_{X} Y$. Thus, we get (1.4).

By assumption $u \in L_{1}^{2}(M, N)$. This ensures that $\partial_{\alpha} u, \alpha=1,2, \ldots, n$, exist almost everywhere. Hence, we come to the conclusion that

$$
\Delta \tilde{u}^{i}(x)=g^{\alpha \beta}(x) \cdot \tilde{A}_{u(x)}^{i}\left(\partial_{\alpha} \tilde{u}, \partial_{\beta} \tilde{u}\right)
$$

weakly.

In the next section we will make some general remarks about the regularity problem of harmonic maps. Section 3 consists of a collection of preliminary results. In the last two sections we study stationary maps whose singular set is of codimenion greater than 2 .

2. Regularity problem of harmonic maps. In this section we want to make precise the concept of regularity of harmonic maps.

A harmonic map $u$ is by our definition a weak solution to a nonlinear elliptic system in local coordinates. Even in the unconstrained case there is no reason to hope that it always will be continuous (cf. [Fr]).

Next we remark that if a harmonic map has small oscillation then it is smooth. This is a well known fact to specialists in this area but had not appeared explicitly in its full scale. It was shown in $[\mathbf{H}, \mathbf{W}]$ that a weakly harmonic map with small oscillation is Hölder continuous. Recently a proof was given in [Sch] to show that if a weakly harmonic map $u$ is Hölder continuous then $\nabla u$ is locally bounded, i.e., $\nabla u \in L_{\text {loc }}^{\infty}$. By the harmonic map system, one then gets $\Delta u \in L_{\text {loc }}^{\infty}$. By the $L^{p}$ theory of linear elliptic systems one deduces that $u \in L_{2, \text { loc }}^{p}$ for $p<\infty$. From the harmonic map system we see that $\Delta u \in L_{1, \text { loc }}^{p}$. Hence, $u \in L_{3, \text { loc }}^{p}$. Repeating this procedure, we get that $u \in L_{k, \text { loc }}^{p}$ for $p<\infty$ and $k=1,2,3, \ldots$. By Sobolev imbedding theorem $u \in C^{\infty}$.

We say that a point $x \in M$ is a regular point if there is a neighborhood $U$ of $x$ such that $x$ is Hölder continuous on $U$. In view of the above remark, we could assume small oscillations in place of Höder continuity as well. Let $\Omega$ be the set of all regular points in $M$. $\Omega$ is an open set. Its complement is called the singular set of $u$, denoted by $\Sigma(u)$. Clearly, $\Sigma(u)$ is a closed set.

3. Preliminary results. As mentioned in the introduction, for stationary maps, we have a monotonicity inequality.

Lemma (3.1) (Monotonicity inequality). Suppose $u$ is a stationary map from $B(O)$ into $N \subset \mathbf{R}^{k} . B(O)$ is the unit ball in $\mathbf{R}^{n}$ equipped with a 
Riemannian metric. For $n>2$ we have for $0<\sigma<\rho<\operatorname{dist}\left(x_{0}, \partial B\right)$

$$
\begin{gathered}
e^{C \Lambda \rho_{2}} \cdot \rho_{2}^{2-n} \cdot \int_{B_{\rho_{2}}\left(x_{0}\right)}|\nabla u|^{2} d V-e^{C \Lambda \rho_{1}} \cdot \rho_{1}^{2-n} \cdot \int_{B_{\rho_{1}}\left(x_{0}\right)}|\nabla u|^{2} d V \\
\geq 2 \int_{B_{\rho_{2}}\left(x_{0}\right) \backslash B_{\rho_{1}}\left(x_{0}\right)} e^{C \Lambda r} \cdot\left|x-x_{0}\right|^{2-n} \cdot\left|\partial_{r} u\right|^{2} d V
\end{gathered}
$$

where $\Lambda$ and $C$ are constants.

For a proof one can read $[\mathbf{P r}]$. This type of inequality gives some useful information about the map $u$. In particular, we see that

$$
\int_{B(O)}\left|x-x_{0}\right|^{2-n} \cdot\left|\partial_{r} u\right|^{2} d V \leq C \cdot E(u)
$$

where $E(u)$ is the total energy of $u$.

The basic a priori estimates used in this work were obtained by $\mathrm{R}$. Schoen and K. Uhlenbeck. We state it here as a Lemma.

Lemma (3.2) [Sch]. Suppose $u \in C^{2}\left(B_{r}^{n}, N\right)$ is harmonic with respect to a metric $g$ on $B_{r}^{n}$. Suppose that

$$
\begin{gathered}
\Lambda^{-1} \cdot\left(\delta_{\alpha \beta}\right) \leq g_{\alpha \beta} \leq \Lambda \cdot\left(\delta_{\alpha \beta}\right), \\
\left|\partial_{\nu} g_{\alpha \beta}\right| \leq \Lambda \cdot r^{-1} .
\end{gathered}
$$

There exists $\varepsilon=\varepsilon(\Lambda, n, N)>0$ such that if

$$
r^{2-n} \cdot \int_{B_{r}}|\nabla u|^{2} d V \leq \varepsilon
$$

then

$$
r^{2} \cdot \sup _{B_{r_{2}}}\left\{|\nabla u|^{2}\right\} \leq C \cdot r^{2-n} \int_{B_{r}}|\nabla u|^{2} d V .
$$

We outline its proof because of its importance in this paper. The proof of this lemma given in [Sch] makes use of Lemma 1, noticing that ' $C^{2}$ harmonic' implies 'stationary', to construct a scaled version $v$ of $u$. The map $v$ satisfies

$$
|\nabla v|^{2}(0)=1, \quad \sup \left\{|\nabla v|^{2}\right\} \leq 4
$$

in a ball $B_{r_{0}}$ with $r_{0}<1$ if the energy of $u$ is small. Then it follows from the Bochner formula that in $B_{r_{0}}$

$$
\Delta\left(|\nabla v|^{2}\right) \geq-C \cdot|\nabla v|^{2}
$$


The conclusion (2.3) is an immediate consequence of the mean value inequality of C. B. Morrey [M, 5.3.1].

In our regularity proof, this lemma is used in the following way. Suppose that a point $x$ is away from the singular set $\Sigma(u)$. Apply the Lemma to $B_{r}(x)$ where $r=\operatorname{dist}(x, \Sigma(u))$. Then

$$
r^{2} \cdot|\nabla u|^{2}(x) \leq C \cdot r^{2-n} \int_{B_{r}}|\nabla u|^{2} d V
$$

In some cases we can bound

$$
C \cdot r^{2-n} \int_{B_{r}}|\nabla u|^{2} d V
$$

by the total energy $E(u)$. Thus we get an a priori estimate of $|\nabla u|^{2}$ :

$$
|\nabla u|^{2} \leq \frac{C E(u)}{r^{2}}
$$

Lemma (3.3). (First variation formula.) For a smooth family $\phi_{t}$ of diffeomorphisms which are the identity near $\partial B$ we let $u_{t}=u \circ \phi_{t}$. We then have

$$
\left.\frac{d}{d t} E\left(u_{t}\right)\right|_{t=0}=-\int_{B}\left[|d u|^{2} \cdot \operatorname{div} X-2\left\langle d u\left(\nabla_{e_{1}} X\right), d u\left(e_{i}\right)\right\rangle\right] d V
$$

where $X=$ the variation vector field $=\left.(d / d t) \varphi_{t}\right|_{t=0}, e_{i}, i=1, \ldots, n$ form an orthonormal basis on $B$.

This is a standard result. One can prove it by a change of coordinates.

We mention the following regularity lemma by C. B. Morrey.

Lemma (3.4). (C. B. Morrey). Suppose $0<\alpha<1$ and $c<\infty$. If $u \in L_{1}^{2}(M, N)$ and

$$
\gamma^{2-n} \int_{B_{\gamma}[x]}|\nabla u|^{2} d x \leq c \gamma^{2 \alpha}
$$

for any $x \in B$ and $\gamma \in\left(0, \frac{1}{4}\right)$, then $u$ is Hölder continuous on $B$.

4. Higher dimensional singular set. In [Liao] it was proved that isolated singular points are removable if the total energy is small. A natural question arises, i.e., what can we say if the apparent singular set has higher dimension? In this and the next sections we will take on this problem and prove our main theorem. 
By rescaling and by taking normal coordinates, we can work on a ball $B$ in $\mathbf{R}^{n}$ with a Riemannian metric $g$, which is almost Euclidean. We prove the following

Proposition (4.1). Suppose for any $K>0$, there exist numbers $\varepsilon_{0}$, $\sigma \in(0,1)$ depending only on the metric $g, K$ and $N$ so that if

(1) $\Sigma \subset$ the graph of a $C^{1, \alpha}$ vector valued function

$$
f: \mathbf{B}^{n} \cap \mathbf{R}^{d} \rightarrow \mathbf{R}^{n}, \quad n-d>2, \quad|f|_{1, \alpha} \leq K,
$$

and

(2) $u \in C^{\infty}(B \backslash \Sigma, N)$ is a stationary map with $E(u) \leq \varepsilon_{0}$, then

$$
\sigma^{2-n} E_{\sigma}(u) \leq \frac{1}{2} E(u),
$$

where $E_{\sigma}(u)=\int_{B_{\sigma}}|\nabla u|^{2} d v, B_{\sigma}$ is the geodesic ball of radius $\sigma$, where $u_{\sigma}$ is defined by $u_{\sigma}(x)=u(\sigma x)$. Thus Proposition (4.1) asserts that

$$
E\left(u_{\sigma}\right) \leq \frac{1}{2} E(u) .
$$

This is an energy improving type of inequality.

We prove it by contradiction.

Proof. Assume that the conclusion is false. Then for $i=1,2,3, \ldots$, there is a stationary map

$$
u_{i} \in C^{\infty}\left(\mathbf{B}^{n} \backslash \Sigma_{i}, N\right),
$$

whose singular set $\Sigma_{i} \subset$ the graph of a $C^{1, \alpha}$-vector valued function $f_{i}$ on $\mathbf{R}^{d},\left|f_{i}\right|_{1, \alpha} \leq K$, such that $E\left(u_{l}\right) \leq 1 / i$ but

$$
\sigma^{2-n} E_{\sigma}\left(u_{i}\right) \geq \frac{1}{2} E\left(u_{i}\right) \text {. }
$$

Define a scaled map $v_{i}$ by

$$
v_{i}=\left(u_{i}-\bar{u}_{i}\right)\left[E\left(u_{i}\right)\right]^{-1 / 2},
$$

where $\bar{u}_{i}$ is the average of $u_{i}$. Note that

$$
E\left(v_{i}\right)=E\left(u_{i}\right) \cdot E\left(u_{i}\right)^{-1}=1 .
$$

By the weak compactness, there is a subsequence (again denoted by $v_{i}$ ) such that (weakly)

$$
v_{i} \rightarrow v_{\infty} \in L_{1}^{2}\left(M, \mathbf{R}^{k}\right) .
$$

Dividing (4.4) by $E\left(u_{i}\right)$, we get

$$
\boldsymbol{\sigma}^{2-n} E_{\boldsymbol{\sigma}}\left(v_{i}\right) \geq \frac{1}{2} .
$$


The harmonic map $u_{i}$ satisfies elliptic system

$$
\Delta u_{i}=g^{\alpha, \beta} A\left(\partial_{\alpha} u_{l}, \partial_{\beta} u_{i}\right),
$$

where $A$ is a quadratic form. Dividing (4.6) by $E\left(u_{l}\right)^{1 / 2}$, we get

$$
\Delta v_{i}=E\left(u_{i}\right)^{1 / 2} \cdot g^{\alpha \beta} \cdot A\left(\partial_{0} v_{i}, \partial_{\beta} v_{i}\right) .
$$

Letting $i \rightarrow \infty$, since $E\left(u_{i}\right) \rightarrow 0$, we get

$$
\Delta v_{\infty}=0 \text {. }
$$

By the Weyl Lemma $v_{\infty}$ is smooth. Our plan is to show

$$
E_{\sigma}\left(v_{l}\right) \rightarrow E_{\sigma}\left(v_{\infty}\right) \text { as } i \rightarrow \infty \text {. }
$$

Let $\eta>0$ be an arbitrary constant. The $C^{1, \alpha}$ boundedness of $f_{t}$ enables us to extract a subsequence (again denoted by $f_{i}$ ) so that $f_{l} \rightarrow f$ uniformly in $C^{1}$ norm. The limit $f$ is $C^{1, \alpha}$. Let the graph of $f$ be $\Sigma$.

$$
\Sigma=\left\{\left(x^{\prime}, f\left(x^{\prime}\right)\right) \in \mathbf{R}^{n}: x^{\prime} \in \mathbf{R}^{d} \cap B\right\} .
$$

Consider the tube neighborhood

$$
\Sigma_{\lambda}=\left\{x \in \mathbf{R}^{n}: \operatorname{dist}(x, \Sigma) \leq \lambda\right\} .
$$

Fix $\lambda_{0}>0$ such that for $\lambda \leq \lambda_{0}$

$$
\int_{B_{\sigma} \cap \Sigma_{\lambda}}\left|\nabla v_{\infty}\right|^{2} \leq \frac{1}{3} \cdot \eta
$$

Cover $\Sigma_{\lambda} \cap B_{\sigma}$ by balls centered at points $x_{j}, j=1,2, \ldots, N(\lambda)$, with radii $\mu=C_{1} \cdot \lambda$. Because the metric $g$ on $B$ is close to the Euclidean metric, we can arrange so that the number of these balls is bounded. More explicitly

$$
N(\lambda) \leq C_{l} \cdot \lambda^{-d}
$$

By the monotonicity inequality

$$
\mu^{2-n} \int_{B_{\mu}\left(x_{j}\right)}\left|\nabla u_{i}\right|^{2} d V \leq C_{3} \cdot E\left(u_{i}\right) .
$$

In terms of $v_{1}$ we have

$$
\int_{B_{\mu}\left(x_{1}\right)}\left|\nabla v_{i}\right|^{2} d V \leq C_{3} \cdot \mu^{n-2} .
$$

From these estimates, we get

$$
\int_{B_{\sigma} \cap \Sigma_{\lambda}}\left|\nabla v_{l}\right|^{2} d V \leq N(\lambda) \cdot \int_{B_{\mu}\left(x_{J}\right)}\left|\nabla v_{l}\right|^{2} d V \leq C_{4} \cdot \lambda^{-d} \cdot \lambda^{n-2} .
$$


By assumption $n-d>2$, we can take $\lambda_{1}>0$ such that

$$
\int_{B_{\sigma} \cap \Sigma_{\lambda}}\left|\nabla v_{i}\right|^{2} d V \leq \frac{1}{3} \eta
$$

for $i=1,2,3, \ldots$ and all $0<\lambda \leq \lambda_{1}$. Fix $\lambda>0$ such that $\lambda \leq$ $\min \left(\lambda_{0}, \lambda_{1}\right)$. There is an integer $I_{1}$ such that

$$
\Sigma_{i} \cap B_{\sigma} \subset \Sigma_{\lambda} \cap B_{\sigma}
$$

if $i \geq I_{1}$. Using the estimate (3.4), we get

$$
\left|\nabla v_{i}\right| \leq C_{5}
$$

on $B_{\sigma} \backslash \Sigma_{\lambda}$. In particular, there is a subsequence (again denoted by $v_{i}$ ) such that

$$
\int_{B_{\sigma} \backslash \Sigma_{\lambda}}\left|\nabla v_{i}\right|^{2} d V \rightarrow \int_{B_{\sigma} \backslash \Sigma_{\lambda}}\left|\nabla v_{\infty}\right|^{2} d V, \quad \text { as } i \rightarrow \infty .
$$

Thus we can take $I_{2}>I_{1}$ so that for $i \geq I_{2}$

$$
\left|\int\left(\left|\nabla v_{i}\right|^{2}-\left|\nabla v_{\infty}\right|^{2}\right) d V\right| \leq \frac{1}{3} \eta \text {. }
$$

From inequalities (4.7), (4.8), and (4.9), we get for $i \geq I_{2}$

$$
\left|\int\left(\left|\nabla v_{i}\right|^{2}-\left|\nabla v_{\infty}\right|^{2}\right) d V\right| \leq \frac{1}{3} \eta+\frac{1}{3} \eta+\frac{1}{3} \eta=\eta .
$$

Hence we have

$$
E_{\sigma}\left(v_{i}\right)-E_{\sigma}\left(v_{\infty}\right) \text { as } i \rightarrow \infty .
$$

In particular, from (4.5) we get

$$
\sigma^{2-n} E_{\sigma}\left(v_{\infty}\right) \geq \frac{1}{2} .
$$

On the other hand we have from linear theory (cf. [M])

$$
\sigma^{2-n} E_{\sigma}\left(v_{\infty}\right) \leq \sigma^{2-n} \sigma^{n} \cdot \sup _{B_{1 / 2}}\left|v_{\infty}\right|^{2} \leq \sigma^{2} \cdot C_{6},
$$

where $C_{6}$ is a universal constant (depending on $n$ ). If we fix $\sigma$ small such that

$$
\sigma^{2}<\frac{1}{2} C_{6}^{-1}
$$

we would have a contradiction to (4.10). Thus the conclusion of the proposition is true.

5. Proof of the main regularity theorem. We proceed to prove our main result that a stationary map $u$, whose singular set $\Sigma$ has codimension at least 2 , is regular if its total energy is small. 
First let us consider a scaling property. Define

$$
u_{\lambda, x_{0}}: B-\mathbf{R}^{k}
$$

by

$$
u_{\lambda, x_{0}}(x)=u\left(x_{0}-\lambda x\right)
$$

as before. We want to show that condition (1) in (4.1) is preserved under scaling. To see this, let

$$
\begin{gathered}
u \in C^{\infty}(B \backslash \Sigma, N), \\
|f|_{1, \alpha} \leq K, \\
\Sigma=\left\{(v, w) \in \mathbf{R}^{n}: v=f(w) \in \mathbf{R}^{n-d}, w \in \mathbf{R}^{d} \cap B\right\} .
\end{gathered}
$$

Let

$$
\Sigma_{\lambda}=\left\{(v, w) \in \mathbf{R}^{n}: v=\lambda^{-1} f(\lambda w), w \in \mathbf{R}^{d} \cap B\right\} .
$$

Suppose $x=(w, v) \in$ the singular set of $u_{\lambda}$. Then

$$
(\lambda w, \lambda v) \in \Sigma \text {. }
$$

Thus $\lambda v=f(\lambda w)$. That is $v=\lambda^{-1} f(\lambda w)$. Hence $x$ must belong to $\Sigma_{\lambda}$. Denoting $\lambda^{-1} \cdot f(\lambda w)$ by $f_{\lambda}(w)$, we get

$$
\begin{aligned}
\nabla f_{\lambda}(w) & =\nabla f(\lambda w), \\
\left|\nabla f_{\lambda}\left(w_{1}\right)-\nabla f_{\lambda}\left(w_{2}\right)\right| & =\left|\nabla f\left(\lambda w_{1}\right)-\nabla f\left(\lambda w_{2}\right)\right| \\
& \leq K\left|\lambda w_{1}-\lambda w_{2}\right|^{\alpha} \leq K \cdot\left|w_{1}-w_{2}\right|^{\alpha}
\end{aligned}
$$

for $0<\lambda<1$. Thus $f_{\lambda}$ is again $C^{1, \alpha}$ and

$$
\left|f_{\lambda}\right|_{1, \alpha} \leq K \text {. }
$$

Next we assume that $u$ satisfies the assumptions of the main theorem. Let $\sigma \in\left(0, \frac{1}{2}\right)$ be given in Proposition (4.1). We write $u_{\sigma}=u_{\sigma, x_{0}}$ for $x_{0} \in \Sigma$. Observe that

$$
E\left(u_{\sigma}\right)=\sigma^{2-n} \cdot E_{\sigma}(u) \leq \frac{1}{2} E(u) .
$$

By the scaling property we remarked above, $u_{\sigma}$ satisfies all conditions in Proposition (4.1). Hence

$$
\sigma^{2-n} E_{\sigma}\left(u_{\sigma}\right) \leq \frac{1}{2} E\left(u_{\sigma}\right) .
$$

We can write this as

$$
\sigma^{2-n} \cdot \sigma^{2-n} \cdot E_{\sigma^{2}}(u) \leq \frac{1}{2} \cdot \frac{1}{2} \cdot E(u),
$$


i.e.,

$$
\left(\sigma^{2}\right)^{2-n} \cdot E_{\sigma^{2}}(u) \leq 2^{-2} \cdot E(u) .
$$

Observe that one can write this inequality as

$$
E\left(u_{\sigma^{2}}\right) \leq 2^{-2} \cdot E(u) .
$$

Again $u_{\sigma^{2}}$ satisfies the conditions in Proposition (4.1). Thus

$$
\sigma^{2-n} E_{\sigma}\left(u_{\sigma^{2}}\right) \leq \frac{1}{2} E\left(u_{\sigma^{2}}\right)
$$

i.e.,

$$
\sigma^{2-n} \cdot\left(\sigma^{2}\right)^{2-n} \cdot E_{\sigma^{3}}(u) \leq \frac{1}{2} \cdot 2^{-2} \cdot E(u) .
$$

Hence

$$
\begin{aligned}
\left(\sigma^{3}\right)^{2-n} \cdot E_{\sigma^{3}}(u) & \leq 2^{-3} \cdot E(u), \\
E\left(u_{\sigma^{3}}\right) & \leq 2^{-3} \cdot E(u) .
\end{aligned}
$$

Repeating this procedure, we get for $i=1,2,3, \ldots$

$$
E\left(u_{\sigma^{\prime}}\right) \leq 2^{-i} \cdot E(u) .
$$

We claim that there is a $\beta>0$ such that for any $r \in(0, \sigma)$

$$
r^{2-n} E_{r}(u) \leq C \cdot r^{\beta} \cdot E(u) .
$$

To see that (5.2) holds, take $\beta=\ln 2 / \ln \left(\sigma^{-1}\right)$ where $\sigma$ is obtained by Proposition (4.1) and is less than $1 / 2$. Given any $r \in(0, \sigma)$, there is an integer $i$ such that

$$
\sigma^{1+l} \leq r \leq \sigma^{l}
$$

We have

$$
\begin{aligned}
\ln \left(\sigma^{-1} r^{\beta}\right) & =\ln \left(\sigma^{-1}\right)+\beta \ln r \geq \ln \left(\sigma^{-1}\right)+\frac{\ln 2}{\ln \left(\sigma^{-1}\right)} \cdot \ln \left(\sigma^{i+1}\right) \\
& \geq \ln \left(\sigma^{-1}\right)+\ln \left(2^{-1}\right)+\ln \left(2^{-i}\right) \\
& \geq-\ln (2 \sigma)+\ln \left(2^{-i}\right) \geq \ln \left(2^{-t}\right)
\end{aligned}
$$

since $0<2 \sigma<1$. Thus

$$
\sigma^{-1} r^{\beta} \geq 2^{-l}
$$

We get from (5.1) that

$$
r^{2-n} E_{r}(u) \leq C_{1} \cdot\left(\sigma^{i}\right)^{2-n} \cdot E_{\sigma^{i}}(u) \leq C_{2} \cdot 2^{-i} E(u) .
$$


By (5.3), we have

$$
r^{2-n} \cdot E_{r}(u) \leq C_{2} \sigma^{-1} \cdot r^{\beta} \cdot E(u) .
$$

Since $\sigma$ is a constant we can take $C=C_{2} \sigma^{-1}$ and (5.2) is proved.

By a theorem of C. B. Morrey (cf. [M]) $u$ is regular on a small ball centered at $x_{0}$. Apply this argument to every point of $\Sigma$. We then have the desired result that $u$ is regular provided its total energy is less than $\varepsilon$ given by the Proposition (4.1). Thus the main theorem has been proved.

Acknowledgment. I greatly appreciate the encouragement and advice from Professor R. Schoen.

\section{REFERENCES}

[E, L] J. Eells and L. Lemaire, A report on harmonic maps, Bull. London Math.

[F] J. Frehse, $A$ discontinuous solution to a mildly nonlinear elliptic system, Math. Z., 134 (1973), 229-230.

[G, R, S, B] W. Barger, S. N. M. Ruijsenaars, E. Seiler and D. Buren, On finite action solutions of the nonlinear o-model, Annals of Physics, 119 (1979), 305-325.

$[\mathrm{H}, \mathrm{W}] \quad \mathrm{S}$. Hildebrandt and K. O. Widman, On the Hölder continuity of weak solutions of quasilinear elliptic systems of second order, Ann. Sci. Norm. Suppisa, IV (1977), 145-178.

[Liao] G. Liao, $A$ regularity theorem for harmonic maps with small energy, J. Differential Geom., 22 (1985), 233-241.

[M] C. B. Morrey, Jr., Multiple integrals in the calculus of variations, (1966), Springer-Verlag, New York,

[P] P. Price, A monotonicity formula for Yang-Mills fields, Manuscripta Math., 43 (1983), 131-166.

[Sa.U] J. Sacks and K. Uhlenbeck, The existence of minimal immersions of 2-spheres, Ann. of Math., 113 (1981), 1-24.

[Sch] R. Schoen, Analytic aspects of the harmonic map problem, Seminar on Nonlinear Partial Differential Equations, ed. S. S. Chern, Springer-Verlag, (1985), 321-358.

[Sch, U] R. Schoen and K. Uhlenbeck, J. Differential Geom., 17 (1982), 307-335.

[Sch.U] _ Regularity of minimizing harmonic maps into the spheres, Invent. Math., 78 (1984), 89-100.

[Kin.Har.Lin] D. Kinderlehrer, R. Hardt and F.-H. Lin, Existence and partial regularity of static liquid crystal configuration, Comm. Math. Physics, 105 (1986), 547-570.

Received September 15, 1986 and in revised form November 21, 1986.

UNIVERISTY OF UTAH

Salt Lake City, UT 84112 


\section{PACIFIC JOURNAL OF MATHEMATICS EDITORS}

\author{
V. S. VARADARAJAN \\ (Managing Editor) \\ University of California \\ Los Angeles, CA 90024 \\ HERBERT ClEMENS \\ University of Utah \\ Salt Lake City, UT 84112 \\ R. FINN \\ Stanford University \\ Stanford, CA 94305
}

\author{
HERMANN FLASCHKA \\ University of Arizona \\ Tucson, AZ 85721
}

RAMESh A. GANGolli

University of Washington Seattle, WA 98195

VAUGHAN F. R. JONES

University of California

Berkeley, CA 94720

\author{
ROBION KIRBY \\ University of California \\ Berkeley, CA 94720
}

C. C. MOORE

University of California

Berkeley, CA 94720

HAROLD STARK

University of California, San Diego

La Jolla, CA 92093

\section{ASSOCIATE EDITORS}
R. AREnS
E. F. BECKENBACH
B. H. NEUMANN
F. WOLF
K. YOSHIDA

(1906-1982)

\section{SUPPORTING INSTITUTIONS}

\begin{abstract}
UNIVERSITY OF ARIZONA
UNIVERSITY OF BRITISH COLUMBIA

CALIFORNIA INSTITUTE OF TECHNOLOGY

UNIVERSITY OF CALIFORNIA

MONTANA STATE UNIVERSITY

UNIVERSITY OF NEVADA, RENO

NEW MEXICO STATE UNIVERSITY

OREGON STATE UNIVERSITY
\end{abstract}

\author{
UNIVERSITY OF OREGON \\ UNIVERSITY OF SOUTHERN CALIFORNIA \\ STANFORD UNIVERSITY \\ UNIVERSITY OF HAWAII \\ UNIVERSITY OF TOKYO \\ UNIVERSITY OF UTAH \\ WASHINGTON STATE UNIVERSITY \\ UNIVERSITY OF WASHINGTON
}

The Supporting Institutions listed above contribute to the cost of publication of this Journal, but they are not owners or publishers and have no responsibility for its content or policies.

Mathematical papers intended for publication in the Pacific Journal of Mathematics should be in typed form or offset-reproduced (not dittoed), double spaced with large margins. Please do not use built up fractions in the text of the manuscript. However, you may use them in the displayed equations. Underline Greek letters in red, German in green, and script in blue. The first paragraph must be capable of being used separately as a synopsis of the entire paper. In particular it should contain no bibliographic references. Please propose a heading for the odd numbered pages of less than 35 characters. Manuscripts, in triplicate, may be sent to any one of the editors. Please classify according to the scheme of Math. Reviews, Index to Vol. 39. Supply name and address of author to whom proofs should be sent. All other communications should be addressed to the managing editor, or Elaine Barth, University of California, Los Angeles, California 90024.

There are page-charges associated with articles appearing in the Pacific Journal of Mathematics. These charges are expected to be paid by the author's University, Government Agency or Company. If the author or authors do not have access to such Institutional support these charges are waived. Single authors will receive $\mathbf{5 0}$ free reprints; joint authors will receive a total of $\mathbf{1 0 0}$ free reprints. Additional copies may be obtained at cost in multiples of 50 .

The Pacific Journal of Mathematics is issued monthly as of January 1966. Regular subscription rate: $\$ 190.00$ a year (5 Vols., 10 issues). Special rate: $\$ 95.00$ a year to individual members of supporting institutions.

Subscriptions, orders for numbers issued in the last three calendar years, and changes of address should be sent to Pacific Journal of Mathematics, P.O. Box 969, Carmel Valley, CA 93924, U.S.A. Old back numbers obtainable from Kraus Periodicals Co., Route 100, Millwood, NY 10546.

The Pacific Journal of Mathematics at P.O. Box 969, Carmel Valley, CA 93924 (ISSN 0030-8730) publishes 5 volumes per year. Application to mail at Second-class postage rates is pending at Carmel Valley, California, and additional mailing offices. Postmaster: send address changes to Pacific Journal of Mathematics, P.O. Box 969, Carmel Valley, CA 93924.

PUBLISHED BY PACIFIC JOURNAL OF MATHEMATICS, A NON-PROFIT CORPORATION Copyright (C) 1988 by Pacific Journal of Mathematics 


\section{Pacific Journal of Mathematics}

\section{Vol. 131, No. 2 December, 1988}

Selman Akbulut and Henry Churchill King, Polynomial equations of immersed surfaces .................................... 209

Alberto Baider and Richard C. Churchill, The Campbell-Hausdorff group and a polar decomposition of graded algebra automorphisms ........2 219

Wayne C. Bell and John William Hagood, Separation properties and exact

Radon-Nikodým derivatives for bounded finitely additive measures . . . 237

Dennis J. Garity, James P. Henderson and David G. Wright, Menger

spaces and inverse limits ...............................249

B. Brent Gordon, Algebraically defined subspaces in the cohomology of a

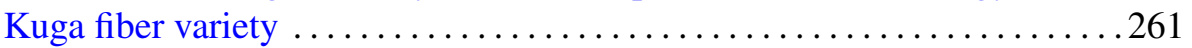

Jeffrey A. Hogan, Weighted norm inequalities for the Fourier transform on connected locally compact groups ........................... 277

Guojun Liao, A study of regularity problem of harmonic maps ..........291

Chin-pi Lu, Modules satisfying ACC on a certain type of colons ......... 303

Kunio Murasugi, Jones polynomials of periodic links

Hans Schoutens, Approximation properties for some non-Noetherian local

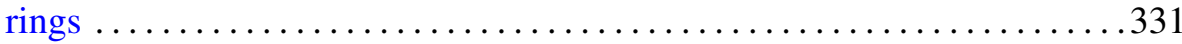

Peter Sjögren, Convergence for the square root of the Poisson kernel ...... 361 Alexandru Ion Suciu, The oriented homotopy type of spun 3-manifolds .393 